\title{
Thermal Convection for Large Prandtl Numbers
}

\author{
Siegfried Grossmann ${ }^{1}$ and Detlef Lohse ${ }^{2}$ \\ ${ }^{1}$ Department of Physics, University of Marburg, Renthof 6, D-35032 Marburg, Germany \\ ${ }^{2}$ Department of Applied Physics and J.M. Burgers Centre for Fluid Dynamics, University of Twente, \\ 7500 AE Enschede, The Netherlands
}

(Received 26 July 2000)

\begin{abstract}
The Rayleigh-Bénard theory by Grossmann and Lohse [J. Fluid Mech. 407, 27 (2000)] is extended towards very large Prandtl numbers Pr. The Nusselt number $\mathrm{Nu}$ is found here to be independent of Pr. However, for fixed Rayleigh numbers $\mathrm{Ra}$ a maximum in the $\mathrm{Nu}(\mathrm{Pr})$ dependence is predicted. We moreover offer the full functional dependences of $\mathrm{Nu}(\mathrm{Ra}, \operatorname{Pr})$ and $\mathrm{Re}(\mathrm{Ra}, \operatorname{Pr})$ within this extended theory, rather than only give the limiting power laws as done in J. Fluid. Mech. 407, 27 (2000). This enables us to more realistically describe the transitions between the various scaling regimes.
\end{abstract}

$$
\begin{gathered}
\epsilon_{u}=\epsilon_{u, \mathrm{BL}}+\epsilon_{u, \text { bulk }}, \\
\epsilon_{\theta}=\epsilon_{\theta, \mathrm{BL}}+\epsilon_{\theta, \text { bulk }} .
\end{gathered}
$$

For the left-hand sides the exact relations $\epsilon_{u}=\frac{\nu^{3}}{L^{4}}(\mathrm{Nu}-$ 1) $\operatorname{RaPr}^{-2}$ and $\epsilon_{\theta}=\kappa \frac{\Delta^{2}}{L^{2}} \mathrm{Nu}$ are used, where $\nu$ is the kinematic viscosity, $\kappa$ the thermal diffusivity, $L$ the height of the cell, and $\Delta$ the temperature differences between bottom and top plates. The local dissipation rates in the $\mathrm{BL}$ and in the bulk [right-hand sides of Eqs. (1) and (2)] are modeled as the corresponding energy input rates, i.e., in terms of $U, \Delta, L$, and the widths $\lambda_{u}$ and $\lambda_{\theta}$ of the kinetic and thermal boundary layers, respectively. For the thickness of the thermal BL we assume $\lambda_{\theta}=L /(2 \mathrm{Nu})$ and for that of the kinetic one $\lambda_{u}=L /(4 \sqrt{\mathrm{Re}})$ as it holds in Blasius type layers [13]; as for the prefactor 1/4 cf. [12], Sec. 3.4. For very large Ra the laminar BL will become turbulent and $\lambda_{u}$ will show a stronger Re dependence. Note that whereas the thermal BLs build up only at the top and bottom wall, the kinetic BL occurs at all walls of the cell and therefore the contribution of $\epsilon_{u, \mathrm{BL}}$ to $\epsilon_{u}$ is larger than a simple minded argument would suggest. The two Eqs. (1) and (2) then allow one to calculate the two dependent variables $\mathrm{Nu}$ and $\mathrm{Re}$ as functions of the two independent ones Ra and Pr.

Input rate modeling. - The modeling of the dissipation rates on the rhs of Eqs. (1) and (2) is guided by the Boussinesq equations. Depending on whether the BL or the bulk contributions are dominant, one gets different expressions on the rhs of Eqs. (1) and (2) and thus different relations for $\mathrm{Nu}, \mathrm{Re}$ vs $\mathrm{Ra}, \mathrm{Pr}$, defining different main regimes; see Ref. [12] and Fig. 1.

The rhs thermal dissipation rates depend on whether the kinetic BL of thickness $\lambda_{u}$ is within the thermal BL of thickness $\lambda_{\theta}\left(\lambda_{u}<\lambda_{\theta}\right.$, small Pr) or vice versa $\left(\lambda_{u}>\right.$ $\lambda_{\theta}$, large $\mathrm{Pr}$ ). The line $\lambda_{u}=\lambda_{\theta}$, corresponding to $\mathrm{Nu}=$ $2 \sqrt{\mathrm{Re}}$, splits the phase diagram into a lower (small Pr) and an upper (large Pr) part, which we label by " $l$ " and " $u$ ".

We first consider $\lambda_{u}<\lambda_{\theta}$ (i.e., small Pr, regime "l"). Then (see [12]) 


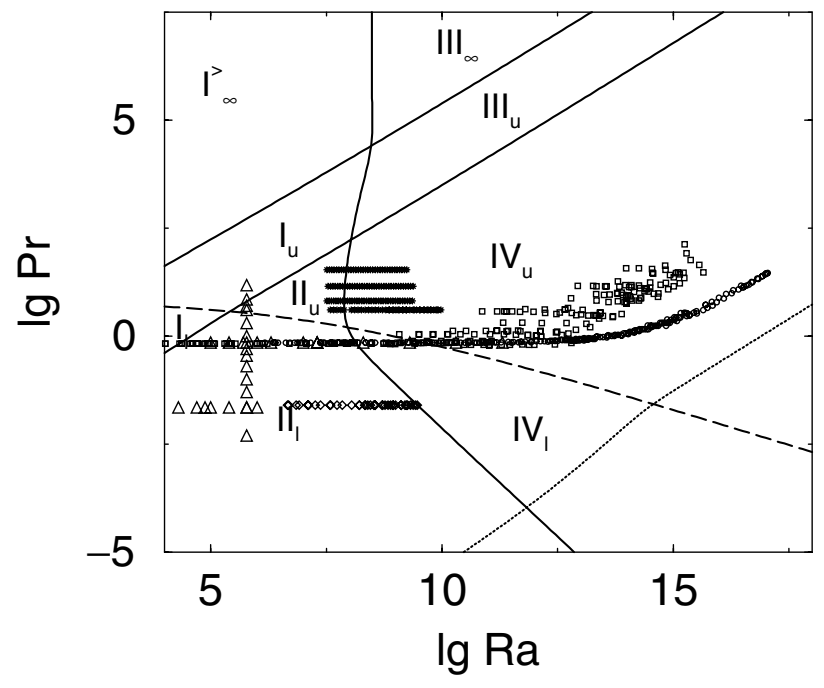

FIG. 1. Phase diagram in the Ra-Pr plane: The upper solid line means $\mathrm{Re}=\operatorname{Re}_{c}(\approx 0.28)$, the lower nearly parallel solid line $\epsilon_{u, \mathrm{BL}}=\epsilon_{u, \mathrm{bulk}}$, the curved solid line is $\epsilon_{\theta, \mathrm{BL}}=\epsilon_{\theta \text {, bulk }}$, and the long-dashed line is the line $\lambda_{u}=\lambda_{\theta}$. The thin dotted line denotes where the laminar kinetic BL becomes turbulent. As extensively discussed in Ref. [12], the exact onset of this instability strongly depends on the prefactors used when calculating this type of phase diagram. Data points where $\mathrm{Nu}$ has been measured or numerically calculated have been included; squares: Chavanne et al. [4]; diamonds: Cioni et al. [3]; circles: Niemela et al. [5]; stars: Xu et al. [7,8]; triangles: Verzicco and Camussi (numerical simulations) [10].

$$
\begin{gathered}
\epsilon_{u, \text { bulk }} \sim \frac{U^{3}}{L} \sim \frac{\nu^{3}}{L^{4}} \operatorname{Re}^{3}, \\
\epsilon_{u, \mathrm{BL}} \sim \nu \frac{U^{2}}{\lambda_{u}^{2}} \frac{\lambda_{u}}{L} \sim \frac{\nu^{3}}{L^{4}} \operatorname{Re}^{5 / 2}, \\
\epsilon_{\theta, \mathrm{bulk}} \sim \frac{U \Delta^{2}}{L} \sim \kappa \frac{\Delta^{2}}{L^{2}} \operatorname{Pr} \operatorname{Re}, \\
\epsilon_{\theta, \mathrm{BL}} \sim \kappa \frac{\Delta^{2}}{L^{2}}(\operatorname{Re} \operatorname{Pr})^{1 / 2} .
\end{gathered}
$$

The last expression is concluded [2,12] from the heat transfer equation $u_{x} \partial_{x} \theta+u_{z} \partial_{z} \theta=\kappa \partial_{z}^{2} \theta$, which implies $U / L \sim \kappa / \lambda_{\theta}^{2}$ giving $\operatorname{Re}^{1 / 2} \operatorname{Pr}^{1 / 2} \sim \mathrm{Nu}$.

If now larger Pr are considered, the kinetic boundary will eventually exceed the thermal one, $\lambda_{u}>\lambda_{\theta}$, upper range " $u$ ". The relevant velocity at the edge between the thermal $\mathrm{BL}$ and the thermal bulk now is less than $U$, namely about $U \lambda_{\theta} / \lambda_{u}$. To describe the transition from $\lambda_{u}$ being smaller to being larger than $\lambda_{\theta}$ we introduce the function $f(x)=\left(1+x^{n}\right)^{-1 / n}$ of the variable $x_{\theta}=$ $\lambda_{u} / \lambda_{\theta}=\mathrm{Nu} / 2 \sqrt{\mathrm{Re}}, f$ being 1 in the lower range "l" (small Pr) and $1 / x_{\theta}$ in " $u$ " (large Pr), respectively. The relevant velocity then is $U f\left(x_{\theta}\right)$. We take $n=4$ to characterize the sharpness of the transition.

This generalizes (5),(6) to

$$
\epsilon_{\theta, \text { bulk }} \sim \kappa \frac{\Delta^{2}}{L^{2}} \operatorname{Pr} \operatorname{Re} f(\mathrm{Nu} / 2 \sqrt{\operatorname{Re}}),
$$

$$
\epsilon_{\theta, \mathrm{BK}} \sim \kappa \frac{\Delta^{2}}{L^{2}} \sqrt{\operatorname{Pr} \operatorname{Re} f(\mathrm{Nu} / 2 \sqrt{\mathrm{Re}})}
$$

while $\epsilon_{u, \text { bulk }}$ and $\epsilon_{u, \mathrm{BL}}$ are still given by (3),(4). Introducing (3),(4),(7),(8) into (1),(2) leads to the Ra,Pr dependences of $\mathrm{Nu}, \mathrm{Re}$ in the upper regime " $u$ ". The pure power laws $\mathrm{Nu}(\mathrm{Ra}, \operatorname{Pr})$ and $\mathrm{Re}(\mathrm{Ra}, \mathrm{Pr})$ in both the lower "l" $\left(\lambda_{u}<\lambda_{\theta}\right.$, small Pr) and the upper " $u$ " $\left(\lambda_{u}>\lambda_{\theta}\right.$, large $\left.\operatorname{Pr}\right)$ regimes are summarized in Table I.

Very large Pr regime. - We now extend the theory to very large Prandtl numbers. As long as $\mathrm{Ra}>\mathrm{Ra}_{c}=1708$ there still is wind, even for very large $\operatorname{Pr}$, as $\mathrm{Ra}_{c}$ is independent of Pr. However, this large scale wind Re slows down with increasing $\operatorname{Pr}$ and eventually becomes laminar throughout the cell. $\lambda_{u}$ can no longer continue to increase according to $\lambda_{u}=L /\left(4 \mathrm{Re}^{1 / 2}\right)$ with decreasing $\mathrm{Re}$, but will saturate to a constant value of order $L$. We call the corresponding Reynolds number $\operatorname{Re}_{c}$ and $\lambda_{u}=L /\left(4 \sqrt{\operatorname{Re}_{c}}\right)$ in this very large Pr regime. To model the smooth transition to the very large Pr regime beyond the line $\mathrm{Re}=\mathrm{Re}_{c}$, i.e., if $\lambda_{u}(\mathrm{Re})=L / 4 \sqrt{\mathrm{Re}}$ approaches $\lambda_{u}\left(\operatorname{Re}_{c}\right)$, we use the crossover function $g(x)=x\left(1+x^{n}\right)^{-1 / n}$ of the crossover variable $x_{L}=\lambda_{u}(\operatorname{Re}) / \lambda_{u}\left(\operatorname{Re}_{c}\right)=\sqrt{\operatorname{Re}_{c} / \operatorname{Re}}$, and again $n=4$. The function $g$ increases linearly, $g\left(x_{L}\right)=x_{L}$, below the transition ( $x_{L}$ small) and is 1 in the very large Pr regime with $\mathrm{Re} \leq \mathrm{Re}_{c}$. In the above modeling for the local dissipation rates we have to replace each $\lambda_{u}$ by $g\left(x_{L}\right) \lambda_{u}\left(\operatorname{Re}_{c}\right)$.

The resulting formulas, given momentarily, will lead, depending on $\mathrm{Ra}$, to three new regimes, valid for very large Pr, denoted as $\mathrm{I}_{\infty}^{<}, \mathrm{I}_{\infty}^{>}$, and $\mathrm{III}_{\infty}$; see Fig. 1 and Table I.

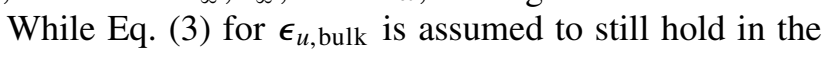
very large Pr range (where $\epsilon_{u}$,bulk hardly contributes to $\epsilon_{u}$ due to the large extension of the kinetic BLs), Eqs. (4)-(6) must be generalized. First generalize (4) for $\epsilon_{u, \mathrm{BL}}$,

$$
\epsilon_{u, \mathrm{BL}} \sim \nu \frac{U^{2}}{g\left(x_{L}\right) L^{2}} \sim \frac{\nu^{3}}{L^{4}} \frac{\mathrm{Re}^{2}}{g\left(\sqrt{\operatorname{Re}_{c} / \mathrm{Re}}\right)} .
$$

Next $\epsilon_{\theta \text {,bulk }}$ : The wind velocity $U$ in (5), which sets the time scale of the stirring, has already been generalized to $U f\left(\lambda_{u} / \lambda_{\theta}\right)$. This equals $U$ itself in the "l" and $U \lambda_{\theta} / \lambda_{u}$ in the " $u$ " regimes. Now, in addition, the explicit $\lambda_{u}$ is to be replaced by $g\left(x_{L}\right) \lambda_{u}\left(\operatorname{Re}_{c}\right)$, i.e.,

$$
\epsilon_{\theta, \text { bulk }} \sim \kappa \frac{\Delta^{2}}{L^{2}} \operatorname{Pr} \operatorname{Re} f\left[\frac{\mathrm{Nu}}{2 \sqrt{\operatorname{Re}_{c}}} g\left(\sqrt{\frac{\operatorname{Re}_{c}}{\operatorname{Re}}}\right)\right] .
$$

Equation (10) simplifies for large enough $\mathrm{Ra}$ (therefore large $f$ argument) and very large $\operatorname{Pr}$ (thus large $g$ argument) to $\epsilon_{\theta \text {, bulk }} \sim \kappa \frac{\Delta^{2}}{L^{2}} \frac{\mathrm{Pr} \mathrm{Re}}{\mathrm{Nu}}$. Inserting (9) and (10) into the rhs of (1) and (2) leads to the new power laws describing the heat flux and the wind velocity in the regime $\mathrm{III}_{\infty}$ beyond $\mathrm{III}_{u}$; cf. Fig. 1,

$$
\mathrm{Nu} \sim \mathrm{Ra}^{1 / 3} \operatorname{Pr}^{0}, \quad \mathrm{Re} \sim \mathrm{Ra}^{2 / 3} \operatorname{Pr}^{-1} .
$$

Finally, $\epsilon_{\theta, \mathrm{BL}}:$ In the thermal boundary layer range beyond $\mathrm{I}_{l}$, relevant for medium Ra, Eq. (6) stays valid, because its derivation did not involve $\lambda_{u}$ and also $f=1$. The 
TABLE I. The pure power laws for $\mathrm{Nu}$ and Re. The prefactors are based on the $c_{i}$ 's given after Eq. (14).

\begin{tabular}{|c|c|c|c|c|}
\hline Regime & Dominance of & BLs & $\mathrm{Nu}$ & $\operatorname{Re}$ \\
\hline $\mathrm{I}_{l}$ & $\boldsymbol{\epsilon}_{u, \mathrm{BL}}, \boldsymbol{\epsilon}_{\theta, \mathrm{BL}}$ & $\lambda_{u}<\lambda_{\theta}$ & $0.22 \mathrm{Ra}^{1 / 4} \operatorname{Pr}^{1 / 8}$ & $0.063 \mathrm{Ra}^{1 / 2} \mathrm{Pr}^{-3 / 4}$ \\
\hline $\mathrm{I}_{u}$ & & $\lambda_{u}>\lambda_{\theta}$ & $0.31 \mathrm{Ra}^{1 / 4} \operatorname{Pr}^{-1 / 12}$ & $0.073 \mathrm{Ra}^{1 / 2} \operatorname{Pr}^{-5 / 6}$ \\
\hline $\mathrm{I}_{\infty}^{\prime \prime}$ & & $\lambda_{u}=L /\left(4 \sqrt{\operatorname{Re}_{c}}\right)>\lambda_{\theta}$ & $0.17 \mathrm{Ra}^{1 / 3}$ & $0.038 \mathrm{Ra}^{2 / 3} \mathrm{Pr}^{-1}$ \\
\hline $\mathrm{I}_{\infty}^{>}$ & & $\lambda_{u}=L /\left(4 \sqrt{\operatorname{Re}_{c}}\right)>\lambda_{\theta}$ & $0.35 \mathrm{Ra}^{1 / 5}$ & $0.054 \mathrm{Ra}^{3 / 5} \mathrm{Pr}^{-1}$ \\
\hline $\mathrm{II}_{l}$ & $\epsilon_{u, \text { bulk }}, \epsilon_{\theta, \mathrm{BL}}$ & $\lambda_{u}<\lambda_{\theta}$ & $0.37 \mathrm{Ra}^{1 / 5} \operatorname{Pr}^{1 / 5}$ & $0.17 \mathrm{Ra}^{2 / 5} \mathrm{Pr}^{-3 / 5}$ \\
\hline $\mathrm{II}_{u}$ & & $\lambda_{u}>\lambda_{\theta}$ & $0.51 \mathrm{Ra}^{1 / 5}$ & $0.19 \mathrm{Ra}^{2 / 5} \operatorname{Pr}^{-2 / 3}$ \\
\hline $\mathrm{III}_{u}$ & $\epsilon_{u, \mathrm{BL}}, \boldsymbol{\epsilon}_{\theta, \text { bulk }}$ & $\lambda_{u}>\lambda_{\theta}$ & $0.018 \mathrm{Ra}^{3 / 7} \operatorname{Pr}^{-1 / 7}$ & $0.023 \mathrm{Ra}^{4 / 7} \mathrm{Pr}^{-6 / 7}$ \\
\hline $\mathrm{III}_{\infty}$ & & $\lambda_{u}=L /\left(4 \sqrt{\operatorname{Re}_{c}}\right)>\lambda_{\theta}$ & $0.027 \mathrm{Ra}^{1 / 3}$ & $0.015 \mathrm{Ra}^{2 / 3} \mathrm{Pr}^{-1}$ \\
\hline $\mathrm{IV}_{l}$ & $\epsilon_{u, \text { bulk }}, \epsilon_{\theta, \text { bulk }}$ & $\lambda_{u}<\lambda_{\theta}$ & $0.0012 \mathrm{Ra}^{1 / 2} \operatorname{Pr}^{1 / 2}$ & $0.025 \mathrm{Ra}^{1 / 2} \mathrm{Pr}^{-1 / 2}$ \\
\hline $\mathrm{IV}_{u}$ & & $\lambda_{u}>\lambda_{\theta}$ & $0.050 \mathrm{Ra}^{1 / 3}$ & $0.088 \mathrm{Ra}^{4 / 9} \mathrm{Pr}^{-2 / 3}$ \\
\hline
\end{tabular}

range $\mathrm{I}_{\infty}^{<}$has to be described by Eqs. (1),(2),(9) (with $g=$ $1)$, and (6), resulting in the same power laws as in regime $\mathrm{III}_{\infty}$, i.e., Eqs. (11). For Pr values above regime $\mathrm{I}_{u}$ Eq. (6) no longer holds. It originated from the heat transport equation. There we now have to use $U f\left(x_{\theta}\right)$ instead of merely $U$. The balance from the heat transfer equation then reads $U f\left(x_{\theta}\right) / L \sim \kappa / \lambda_{\theta}^{2}$. In the $f$ argument $x_{\theta}=\lambda_{u} / \lambda_{\theta}$, the kinetic BL width $\lambda_{u}$ and therefore the crossover function $g$ appears, leading to $\operatorname{Pr} \operatorname{Re} f\left[\frac{\mathrm{Nu}}{2 \sqrt{\operatorname{Re}_{c}}} g\left(\sqrt{\frac{\mathrm{Re}_{c}}{\operatorname{Re}_{e}}}\right)\right] \sim \mathrm{Nu}^{2}$. For very large $\operatorname{Pr}$ [where $g\left(x_{L}\right)=1$ ] above $\mathrm{I}_{u}$ [where $f\left(x_{\theta}\right)=$ $x_{\theta}^{-1}$ ] one obtains the relation $(\operatorname{Re} \operatorname{Pr})^{1 / 3} \sim \mathrm{Nu}$, valid in $\mathrm{I}_{\infty}^{>}$. Together with (1), (2), and (9) one derives the new scaling laws in the interior of $\mathrm{I}_{\infty}^{>}$,

$$
\mathrm{Nu} \sim \mathrm{Ra}^{1 / 5} \operatorname{Pr}^{0}, \quad \mathrm{Re} \sim \mathrm{Ra}^{3 / 5} \operatorname{Pr}^{-1} .
$$

The scaling behavior $\mathrm{Nu} \sim \mathrm{Ra}^{1 / 5}$ has earlier been suggested by Roberts [14]. Note that in all three very large Pr regimes $\mathrm{Nu}$ does not depend on Pr. Furthermore, the upper bound $\mathrm{Nu} \leq$ const $\mathrm{Ra}^{1 / 3}(1+\log \mathrm{Ra})^{2 / 3}$, holding in the limit $\operatorname{Pr} \rightarrow \infty$, is strictly fulfilled [15].

$\mathrm{Nu}$ and $\mathrm{Re}$ in the whole parameter plane.-Plugging now the generalized expressions for the local dissipation rates into the balance Eqs. (1) and (2) finally results in

$$
\begin{aligned}
& \mathrm{Nu} \operatorname{RaPr}^{-2}=c_{1} \frac{\mathrm{Re}^{2}}{g\left(\sqrt{\operatorname{Re}_{c} / \mathrm{Re}}\right)}+c_{2} \operatorname{Re}^{3}, \\
& \mathrm{Nu}=c_{3} \operatorname{Re}^{1 / 2} \operatorname{Pr}^{1 / 2}\left\{f\left[\frac{\mathrm{Nu}}{2 \sqrt{\mathrm{Re}_{c}}} g\left(\sqrt{\frac{\mathrm{Re}_{c}}{\mathrm{Re}}}\right)\right]\right\}^{1 / 2} \\
& +c_{4} \operatorname{Pr} \operatorname{Re} f\left[\frac{\mathrm{Nu}}{2 \sqrt{\operatorname{Re}_{c}}} g\left(\sqrt{\frac{\operatorname{Re}_{c}}{\operatorname{Re}}}\right)\right] \text {. }
\end{aligned}
$$

Here we have added the dimensionless prefactors where appropriate to complete the modeling of the dissipation rates. We have adopted them by a nonlinear fit with the Levenberg-Marquardt method [16] to 151 experimental data points $\mathrm{Nu}(\mathrm{Ra}, \mathrm{Pr})$ obtained by Ahlers [8]; see inset of Fig. $2 \mathrm{~b}$ for the quality of the fit. The result for aspect ratio $\Gamma=1$ is $c_{1}=120, c_{2}=74, c_{3}=0.89, c_{4}=0.048$, and $\operatorname{Re}_{c}=0.28$, and all plots in this paper are based on these numbers. Note that the $c_{i}$ and $\operatorname{Re}_{c}$ may depend on the aspect ratio and are not universal.
The set of Eqs. (13) and (14) is the second main result of this paper. It allows one to calculate $\mathrm{Nu}(\mathrm{Ra}, \mathrm{Pr})$ and $\operatorname{Re}(\mathrm{Ra}, \mathrm{Pr})$ in the whole Ra-Pr parameter space, including all crossovers from any regime to any neighboring one.

All limiting, pure scaling regimes which can be derived from Eqs. (13) and (14) are listed in Table I. The corresponding phase diagram is shown in Fig. 1, completing that of [12] towards very large Pr. Though in the phase diagram we have drawn lines to indicate transitions between the regimes, we note that the crossovers are nothing at all but sharp. All transitions are smeared out of broad ranges. This holds the more, the more similar the scaling exponents of the neighboring regimes are.

Discussion of $\mathrm{Nu}(\mathrm{Pr})$ and $\mathrm{Nu}(\mathrm{Ra})$. - The functions $\mathrm{Nu}\left(\mathrm{Pr}\right.$ ) (for fixed values of $\mathrm{Ra}$ ) and $\mathrm{Nu}(\mathrm{Ra}) / \mathrm{Ra}^{1 / 4}$ (for fixed values of Pr) resulting from Eqs. (13) and (14) are shown in Fig. 2. Nu saturates with increasing Pr. For moderate $\mathrm{Ra}=10^{6}-10^{8}$ a maximum in $\mathrm{Nu}(\mathrm{Pr})$, resulting from the $\mathrm{Nu}$ decrease in regimes $\mathrm{I}_{u}$ and $\mathrm{III}_{u}$, can be seen. It broadens to a plateau for larger $\mathrm{Ra}$, due to the lacking $\mathrm{Pr}$ dependence of $\mathrm{Nu}$ in regime $\mathrm{IV}_{u}$. The decrease in regime $\mathrm{III}_{u}$ from the plateau to the large Pr saturation regime is shifted to larger Pr with increasing Ra, as suggested by Fig. 1.

Figure $2 \mathrm{~b}$ highlights the effects of having finite transition widths. For example, in regime $\mathrm{II}_{l}$ with $\mathrm{Nu} \sim \mathrm{Ra}^{1 / 5}$ (for fixed Pr) the corresponding scaling exponent $1 / 5-$ $1 / 4=-1 / 20$ becomes observable only for very small $\operatorname{Pr} \approx 10^{-3}-10^{-4}$. At $\operatorname{Pr}=10^{-1}$ the roughly four decades of regime $\mathrm{II}_{l}$ (see Fig. 1) between regime $\mathrm{I}_{l}$ and $\mathrm{IV}_{l}$, which both have a stronger $\mathrm{Ra}$ dependence of $\mathrm{Nu}$, are not sufficient to reveal the scaling exponent. And at $\mathrm{Pr}=10^{0}$ the roughly 2.5 decades of regime $\mathrm{II}_{l}$ are nowhere sufficient to lead to a local scaling exponent $d \operatorname{lg~Nu} / d \lg \mathrm{Ra}$ smaller than $1 / 4$.

Similarly, for $\operatorname{Pr}=1$ only for very large $\operatorname{Ra} \gtrsim 10^{14}$ pure scaling $\mathrm{Nu} \sim \mathrm{Ra}^{1 / 3}$ is revealed. For smaller Ra regimes $\mathrm{I}_{u}$ and $\mathrm{I}_{l}$ with $\mathrm{Nu} \sim \mathrm{Ra}^{1 / 4}$ and $\mathrm{II}_{u}$ with $\mathrm{Nu} \sim \mathrm{Ra}^{1 / 5}$ strongly contribute, resulting in an effective local scaling exponent (increasing with Ra) in the range between 0.28 ("2/7") and 0.31 , just as observed in experiment $[1,2,4,5]$.

Comparison to more experiments. - In experiment, it is hard to vary either Ra or Pr over many decades and at 

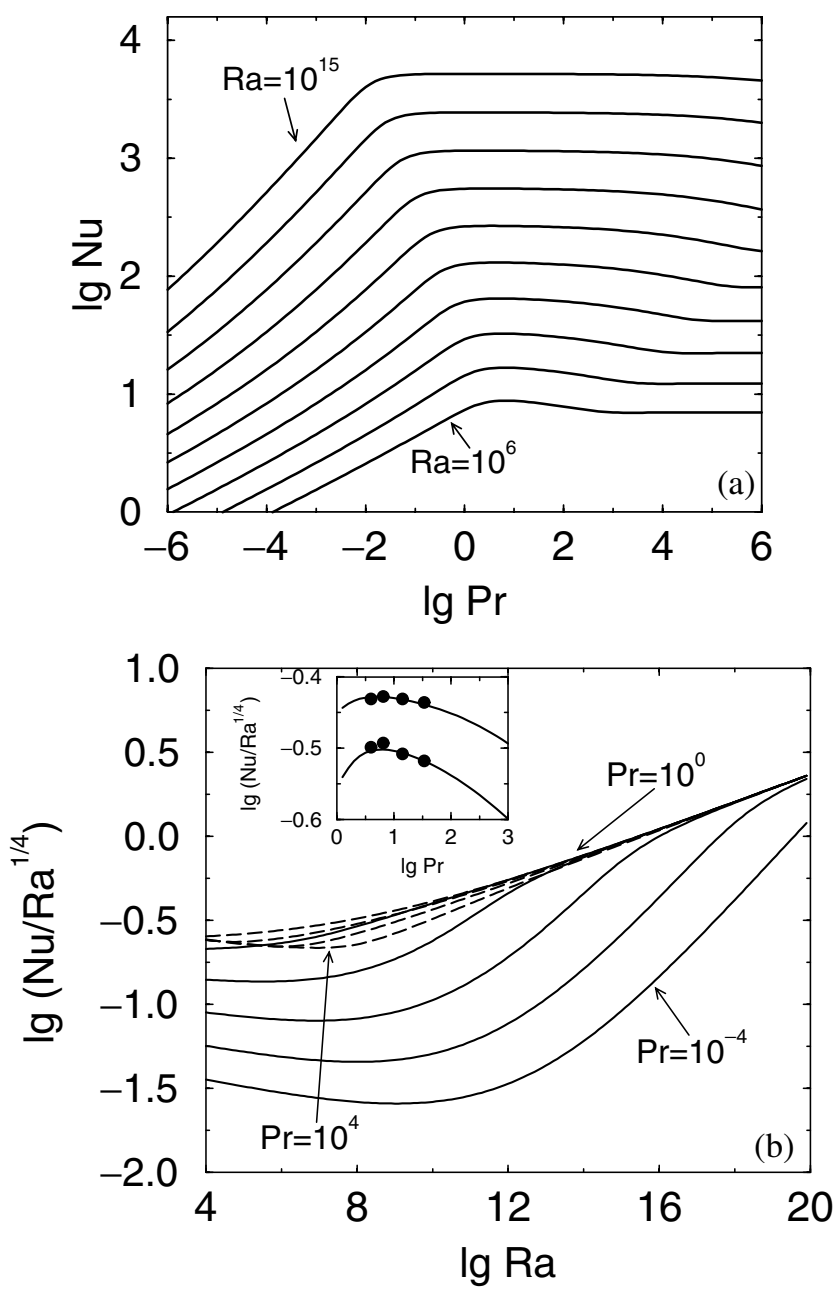

FIG. 2. (a) $\mathrm{Nu}$ as a function of Pr according to theory for $\mathrm{Ra}=$ $10^{6}, \mathrm{Ra}=10^{7}, \ldots$ to $\mathrm{Ra}=10^{15}$, bottom to top. (b) $\mathrm{Nu} / \mathrm{Ra}^{1 / 4} \mathrm{vs}$ Ra for $\operatorname{Pr}=10^{-4}, \ldots, \operatorname{Pr}=10^{0}$ (solid lines, bottom to top) and for $\operatorname{Pr}=10^{1}, \operatorname{Pr}=10^{2}, \operatorname{Pr}=10^{3}$, and $\operatorname{Pr}=10^{4}$ (dashed lines, top to bottom). The inset shows how well the data of Ref. [8] can be fitted within this theory. $\mathrm{Ra}=10^{9.25}$ and $\mathrm{Ra}=10^{7.25}$ for the upper and lower curve, respectively.

the same time to keep the other variable fixed. So most measurements are done along curved lines in the phase space Fig. 1, mixing the $\mathrm{Ra}$ and $\mathrm{Pr}$ dependences. Now Eqs. (13) and (14) allow one to calculate $\mathrm{Nu}$ and $\mathrm{Re}$ along such a curve $\operatorname{Pr}(\mathrm{Ra})$. The present theory suggests that inspite of the different Pr numbers the Nusselt number in the Chavanne et al. experiment [4] should be nearly indistinguishable (due to the lacking Pr dependence of $\mathrm{Nu}$ in regime $\mathrm{IV}_{u}$ ) to that in the Niemela et al. experiment [5], in contrast to what is found; see Fig. 3. Possibly, different temperature boundary conditions have been applied in the two experiments.

This research work was prompted by the experiments of G. Ahlers [8], presented to us at the ITP-Workshop in Santa Barbara in March 2000. We thank him for sharing his results with us prior to publication and for performing the side wall corrections. We also thank him and K. R. Sreenivasan for continuous discussions.

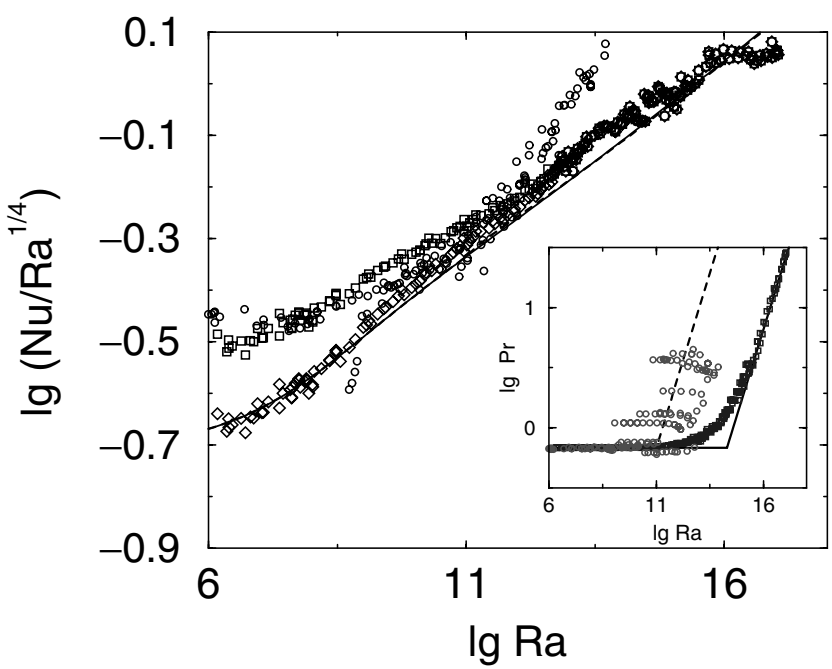

FIG. 3. $\mathrm{Nu}[\mathrm{Ra}, \operatorname{Pr}(\mathrm{Ra})] / \mathrm{Ra}^{1 / 4}$ along the curve $\operatorname{Pr}(\mathrm{Ra}$ ) (shown as boxes in the inset) given by the experimental restrictions of in the Niemela et al. experiment [5]. The diamonds show the side-wall-corrected (following Ref. [17]) data by Niemela et al., the solid line shows the result of the present theory (whose parameters had been adopted to data [8] in a different parameter regime; see text). The boxes show the Niemela et al. data without sidewall corrections, and the circles show the data from the Chavanne et al. experiment [4] (also without sidewall corrections), which were taken along the dashed line in the inset. The Nusselt number along that line is practically indistinguishable from that one along the solid line.

[1] B. Castaing et al., J. Fluid Mech. 204, 1 (1989).

[2] E. D. Siggia, Annu. Rev. Fluid Mech. 26, 137 (1994).

[3] S. Cioni, S. Ciliberto, and J. Sommeria, J. Fluid Mech. 335, 111 (1997).

[4] X. Chavanne et al., Phys. Rev. Lett. 79, 3648 (1997).

[5] J. Niemela, L. Skrebek, K. R. Sreenivasan, and R. Donelly, Nature (London) 404, 837 (2000).

[6] S. Ashkenazi and V. Steinberg, Phys. Rev. Lett. 83, 3641 (1999).

[7] X. Xu, K. M.S. Bajaj, and G. Ahlers, Phys. Rev. Lett. 84, 4357 (2000).

[8] G. Ahlers and X. Xu, following Letter, Phys. Rev. Lett. 86, 3320 (2001).

[9] The experiments reported in [6] suggest a stronger decreasing Nusselt number with increasing Pr, namely $\mathrm{Nu}=0.22 \mathrm{Ra}^{0.3 \pm 0.03} \mathrm{Pr}^{-0.2 \pm 0.04}$ in $10^{9} \leq \mathrm{Ra} \leq 10^{14}$ and $1 \leq \operatorname{Pr} \leq 93$.

[10] R. Verzicco and R. Camussi, J. Fluid Mech. 383, 55 (1999).

[11] R. Kerr and J. Herring, J. Fluid Mech. 419, 325 (2000).

[12] S. Grossmann and D. Lohse, J. Fluid Mech. 407, 27 (2000).

[13] L.D. Landau and E. M. Lifshitz, Fluid Mechanics (Pergamon Press, Oxford, 1987).

[14] G. O. Roberts, Geophys. Astrophys. Fluid Dyn. 12, 235 (1979).

[15] P. Constantin and C. Doering, J. Stat. Phys. 94, 159 (1999).

[16] W. Press et al., Numerical Recipes (Cambridge University Press, Cambridge, 1986).

[17] G. Ahlers, Phys. Rev. E 63, 015303 (2000). 